\title{
Accurate Event Detection and Velocity Estimation in Wireless Environments
}

\author{
Falk Brockmann, Sascha Jungen, Chia-Yen Shih, Marcus Handte and Pedro José Marrón \\ University of Duisburg-Essen, \\ Schützenbahn 70, 45127 Essen, Germany \\ Email: see http://www.nes.uni-due.de/staff/
}

\begin{abstract}
Radio signals can be used to detect the presence of a person (target) in an environment by analysing the fluctuations in the Received Signal Strength Indicator (RSSI). The velocity of the target can be estimated by examining the sequence of disturbances in consecutive radio links over a period of time. This requires knowledge of the deployment of the radio transceivers and the time when the target crosses the Line of Sight (LoS) of each radio link. However, it is not trivial to precisely estimate the exact time of the link crossing due to the broad range of RSSI fluctuations generated as the target approaches the link. In this paper, we evaluate and compare 15 techniques for estimating the velocity of the target and propose enhancements to some of the techniques. In our experiments the techniques perform with an average accuracy in the range between $13.02 \%$ and $96.18 \%$, which corresponds to an average error of $0.05 \mathrm{~m} / \mathrm{s}$ for a moving target.
\end{abstract}

\section{INTRODUCTION}

W IRELESS sensor networks (WSN) have been the interest of the research community for many years. They consist of a number of small, affordable devices (motes), typically equipped with a microprocessor, some sensors, for example a light or humidity sensor, and a transceiver chip for radio transmissions. Traditionally, WSNs are used to monitor environments for long-term changes in attributes like temperature, but recent studies have shown, that they can also be used to detect the presence of persons by analysing disturbances in the radio links between the motes [1], [2], [3], [4], [5].

Generally, if a person (the target) enters a radio link, the human body causes multipath fading of the radio signal [2]. This is detected by analysing for example the Received Signal Strength Indicator (RSSI) of the transmission. The resulting characteristic of the RSSI values will show the presence of a target, but can take different shapes. A highly sensitive link will react early to the presence of a human. The collected RSSI samples will have strongly varying values in a relatively large time interval, even when the target is still some distance away. On the other hand human presence might only create a few higher or lower spikes than the average RSSI signal on an insensitive link. Furthermore, if the target is not just standing still, but moving through the link, this will cause additional fluctuations in the RSSI values [6].

In addition to simply detecting a target it is also possible to estimate the targets' position. One approach would be employing a grid of motes, creating a mesh of multiple radio links [3]. If events are simultaneously detected on several

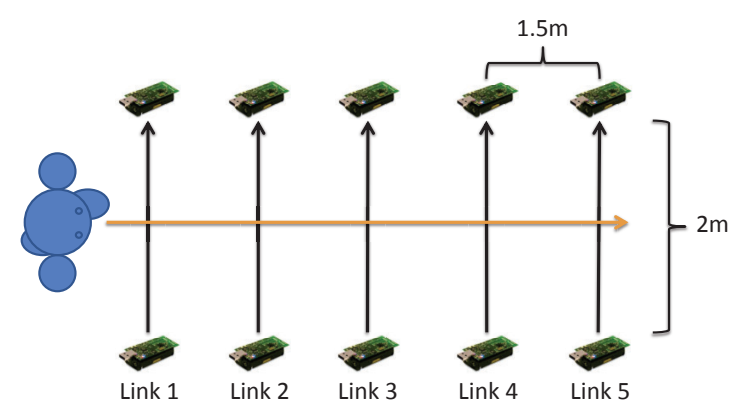

Fig. 1. Concept diagram of experiment set-up

intersecting links, the targets' position can be estimated at this intersection. To track a target through such a system, the detection can be repeated periodically, each time updating the targets' position. Using multiple position estimates it is also possible to derive further information about the targets' behaviour, for example the targets' trajectory or velocity.

There are various reasons why determining the velocity of a target can be useful. For example in a system tracking not one but multiple targets, like in [7], [8], [9], it is beneficial to not only know the position of every target, but also to predict future positions. This information can be used to differentiate between two targets crossing their paths in close vicinity [9]. Knowing the direction and speed is also interesting when monitoring an area where only a limited amount of sensors is available. Since the coverage might be too sparse to allow continuous tracking, a general idea about the movement could be helpful, especially when monitoring an area with movement restrictions. For example, if monitored targets are moving towards a dangerous location, like a broken elevator shaft or the site of a fire, then an alarm sound could be triggered to prevent possible harm.

In any case, the challenge of deducing additional information from the pure detection of a target lies in pinpointing a precise moment within the detection event. This moment should be identifiable, even when the radio link behaviour is different. For our experiments we use the point of the detection event when the target has the greatest influence on the RSSI, which we define as the peak. Since humans are three 


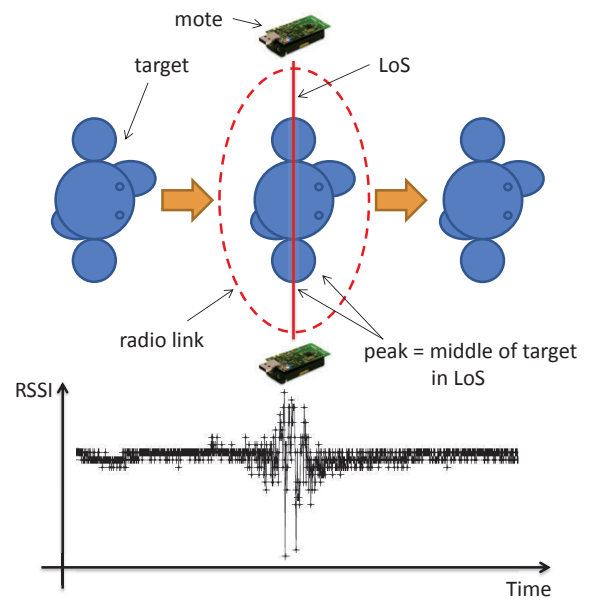

Fig. 2. Target passing the Line of Sight of a radio link

dimensional, it takes a certain time for a target to pass a radio link, as seen in Fig. 2. The peak can be seen as the point in time, when the middle of the target is in the Line of Sight of the link [4]. The goal of this paper is to evaluate 15 different techniques on how to best detect the velocity of a target with the highest possible accuracy. For this we estimate the peak of the radio link crossing event and use the generated results in the velocity estimation of a target moving at different speeds.

Consider the velocity estimation following the simple formula $v=\frac{s}{t}$ as an example. To compute the speed of a target, the locations of at least two radio links are required, and the RSSI values and their respective timestamps are taken from the links. If a target consecutively moves through the radio links, as for example seen in Fig. 1, the actual crossing of a link is recorded as an interval of collected RSSI samples while the link was disturbed. The detection events on both links need to be identified to estimate the time it took the target to cross the distance between the links. Because of the unsteady nature of the RSSI it is not possible to simply use the earliest distorted sample, since its occurrence can be different for dissimilar links. For accurate velocity estimation we have to identify the targets position in the link, respectively for each link. However, the best samples to indicate the actual crossing do not necessarily need to be the samples with the highest RSSI attenuation or the middle sample of the collected interval. The different link states and general fluctuation in the RSSI distort the collected data and make accurate predictions difficult.

The remainder of this paper is structured as follows: Section II gives an overview of research similar to our approach. Section III describes the different techniques used, followed by an evaluation which details the results of our experiments in Section IV. The paper is concluded in Section V with a summary and a short outlook.

\section{RELATED WORK}

Traditionally, instead of using the RSSI to record an event, a system detecting the presence of a target can be based on using a laser range finder. A range finder emits a laser beam that is reflected off of any object it hits. The reflection is caught by the light sensor of the device, the Time of Flight is measured, and the distance to the object computed. If the distance suddenly decreases, the laser beam is reflected at a shorter distance. This is the case, if a target is in the LoS between the laser emitter and the light sensor. However, using a range finder is unfavourable in scenarios, where a larger region needs to be covered, since the laser beam only stretches over a relatively narrow area. Furthermore, the sensor measuring the light intensity needs to be shielded from direct sunlight, to avoid false negatives. Also, these systems can easily be fooled, when a target is avoiding the beam by stepping over it, without interrupting it. Depending on the scenario, a RSSIbased device-free localisation system might be more suitable. Since the RSSI is already disturbed when a target moves near the radio link, avoiding it is not easily possible.

Multiple systems employing the RSSI to detect the presence of a target have been developed [2], [10], [11]. In these systems sensor nodes are deployed at all edges of the monitored area and a grid of radio links is created. A target standing or walking through the grid will cause a characteristic disturbance which can be detected [2].

However, the link characteristic is not specific to a target. In [7] multiple targets are tracked by dividing the monitored area into voxels. A target entering the area will cause disturbances in a set of voxel simultaneously, which are then clustered. Each cluster in the area symbolises a target and is tracked while in the system. But intersecting trajectories create the difficulty of continuously keeping track which target is matched to a specific cluster.

To cope with RSSI irregularities, alternatives to the use of the absolute RSSI or its average have been explored. In [8] the time of flight of radio messages is used in an antenna set-up with five transmit antennas and five receive antennas. The signals reflecting off of the human body are analysed, which allows for the tracking of up to five people. While no longer based on the absolute RSSI, like in [7] still no clear identification of the tracked target out of a group is possible.

The behaviour of the radio links is further analysed in [12]. In general, RSSI values are unstable and always slightly fluctuating because of minimal changes in the environment. Slight variations in the area, for example moving a chair or opening a window, can have a huge impact on the radio propagation. Despite that, [12] defines two classes of observed link behaviour when a target enters the link. Both are based on the different qualities of a link. An anti-fade link has very high RSSI values and will experience a strong attenuation and a significant drop in the RSSI values when a target enters the area of the link. A deep-fade link on the other hand already has a very weak signal quality. For such links it is possible that the quality increases when a target enters the link, due 
to refraction and scattering of the signal. Thus, the resulting RSSI characteristic is generally an inverted version of the antifade one. For this reason and other environmental influences, a detection event can have a very dissimilar RSSI characteristic compared to the characteristic of an event on a different link.

The authors of [4] suggest focusing on the changes in the variance of the RSSI, instead of looking at the absolute value. Regardless whether the absolute RSSI average in- or decreases after a target enters the area of the link, the RSSI variance will always be higher, because the link gets disturbed. Fundamentally, a long-term and a short term variance are computed, compared, and incorporated to the alert magnitude. The larger the alert magnitude is, the more likely a target is present in the detection area. This method simultaneously reduces the influence of different link qualities but also of changes in the environment on the RSSI in long-term measurements. By adapting the long-term variance in phases of tranquillity, in which the variance of the RSSI is low, small changes in the radio propagation model caused by the environment are handled.

Using the RSSI to detect the speed of driving vehicles, [13] collects samples of the signal strength of four links covering a road section. The resulting measurements are analysed using a statistical and a curve fitting technique. In the statistical approach, the time between the entering and exiting event of the monitored area is used to estimate the speed. The curve fitting technique exploits the relation of the height of the variance and the speed of a car. The faster the car passes the monitored area, the higher is the influence on the recorded RSSI variance. However, this technique is applied to fast moving cars made of metal [14], not to humans.

\section{ANALYSED TECHNIQUES}

In this paper we analyse 15 different techniques for estimating the velocity of a target. The techniques are divided into three classes based on their input data. The techniques of the first class are working with the RSSI. They either use all available samples or search for the samples with the minimum value, as introduced in [15]. The techniques of the second class use the alert magnitude, the result of the RSSI variance based algorithm introduced in [4]. The techniques either select the maximum alert magnitude values or again use all available samples. We propose enhancements to the second class to form the third class of techniques. Instead of only using samples with the maximum value, more samples with high values are included into the input data. We evaluate the different methods for estimating the targets' speed in an experiment.

To accurately compute the targets' speed two things are needed: The distance travelled and the time it takes the target to do so. This can be measured for example in a system, where the crossing of the Line of Sight (LoS) between two sensor nodes creates a disturbance in the RSSI values of periodically sent messages. Two measures of the targets' position at two different times can be subtracted from each other to determine the distance. Since the motes in our experiments are set at fixed positions, in the evaluation we consider the distance between them as known. To determine the time, the presence of the target at each position needs to be detected and the detection event analysed. The event itself stretches over an interval consisting of several recorded RSSI samples. Each sample includes a RSSI value and a timestamp. During the recording interval, the target approaches the link, enters it, passes the Line of Sight, and leaves it again. Disturbances in the stream of RSSI values are caused during the time the target stays in the area of the radio link.

Would at this point a sensor producing a boolean result be deployed to record the disturbances, the outcome would be binary: Either a body part of the target is perceived by the sensor and the target is detected, or the sensor is not triggered and no detection is registered. However, the human movement also incorporates the swinging of arms and legs. Therefore, an event can be triggered prematurely, when for example an arm is detected before the rest of the body enters the monitored area, artificially prolonging the event. The targets' distance to the LoS of the radio link will be shifted during the whole event, when compared to the event of a different link. Also, it cannot be assumed that the LoS crossing of the target is in-line with the middle of the detection event. This reduces the accuracy of the velocity estimation.

Fortunately, in the case of detecting an event using RSSI values, the attenuation following the disturbance of the radio link is the highest when the target is closest to the LoS [16]. However, this does not necessarily make the lowest RSSI value the best point describing the event. There might be more than one recorded RSSI sample with the lowest value. Additionally, the RSSI values of a disturbed link are fluctuating. Another sample could time-wise better describe the link crossing, but may not match the lowest RSSI recorded.

The following sections illustrate the techniques to cope with these restrictions and estimate the velocity of a target moving through a group of radio links. For a better overview, all introduced techniques are listed in Table I. Afterwards, the methods are experimentally evaluated.

\section{A. RSSI-based Techniques}

The RSSI-based techniques use the unaltered data stream of RSSI values as input to estimate the events' peak and the targets' velocity. They can be found in the first column of Table I.

1) minRSSI: The first approach to identify the best RSSI sample indicating the peak is the naïve approach, abbreviated minRSSI. This appraoch was also used in [15]. The RSSI data stream of the monitored link is searched for the sample with the minimum value. If multiple samples with the same lowest value are found, all of them are collected. After the time interval of the event has been analysed, the timestamp of the first occurring sample with the lowest RSSI value is selected for the velocity estimation. This approach is easy to implement and low on computational and space complexity, since the samples can be discarded once analysed.

2) medianRSSI: The next approach, medianRSSI, does not simply collect all samples with the minimum value to select 
TABLE I

APPLIED TECHNIQUES ORDERED BY INPUT DATA

\begin{tabular}{|c|c|c|c|}
\hline \multirow[b]{2}{*}{ Technique } & \multicolumn{3}{|c|}{ Based on Value } \\
\hline & Minimum RSSI & Maximum Alert Magnitude & Maximum Alert Magnitude Set \\
\hline Raw & $\operatorname{minRSSI}[15]$ & $\max A l e r t M_{[4],[13]}$ & topMaxAlertM \\
\hline Median & medianRSSI & medianAlertM & topMedianAlertM \\
\hline Average & avgRSSI & avgAlertM & topAvgAlertM \\
\hline Linear Regression & linRegRSSI & linRegAlertM & topLinRegAlertM \\
\hline Curve Fitting & CurveFittingRSSI & CurveFitting [13] & - \\
\hline Cross Correlation & CrossCorrelationRSSI & CrossCorrelation & - \\
\hline
\end{tabular}

the first one, but uses the timestamp of the middle sample. This change is introduced as a precaution against outliers and incorporates additional information about the order in which the samples arrive, the data stream. However, this approach ignores the timestamps of the other collected samples, which might have a beneficial influence on the peak estimation, especially when only two samples with the minimum value exist.

3) avgRSSI: Simply selecting the first sample or selecting the median sample is not always the best choice, given the fact, that an event can have a non-symmetrical characteristic. The avgRSSI approach is addressing this issue by removing the dependency on the precise timestamp of the RSSI sample. Again, all samples with the minimum RSSI value are collected, but then the average of all available timestamps is computed. The new value does not necessarily need to match the timestamp of any sample, but can take an arbitrary point in the event interval. Since the peak does not need to match the sample with the minimum value, or more precisely any single sample, this technique avoids the very limiting dependency of selecting a timestamp from a predefined set.

4) LinRegRSSI: An alternative method, which is also not bound to the exact timestamp of a sample, is the LinRegRSSI approach. LinRegRSSI models the relationship between the detected events on different links by computing a linear regression, based on the RSSI values. The input data set for this approach is the distance between the links and the timestamps of the selected samples. To obtain the necessary data, first all samples with the minimum RSSI value as well as their timestamps are collected for each link. With the distance between the links known in our scenario, the parameters of the linear regression are then estimated from the input.

The simple equation $y=m \cdot x+b$ is the equation of a linear function, where $m$ describes the slope, $b$ the y-intercept.The result of the linear regression is the linear function with the minimum squared error towards all input data points.

In order to estimate the velocity, data from at least two links is needed, but can be extended onto more. In our case, values from all available links are used. The slope of the resulting linear regression line equals the speed of the moving target. To get the time of the peak, the position of a link can be set as $y$ and the equation then solved for $x$.

Ignoring the restrictions of having to choose a precise timestamp, this approach additionally uses information from multiple links.

5) CurveFittingRSSI: A technique introduced in [13] is the fitting of a curve to the data stream of the RSSI variance. The highest point of the curve would indicate the time of the peak. To analyse how well this performs based on the unaltered RSSI stream, we use the CurveFittingRSSI approach. A Gaussian function is fit as continuous curve to the stream of all RSSI values per link. The maximum of the resulting curve is defined by the characteristic of the detection event and will be shifted towards the highest disturbance of the link. Unfortunately this approach is rather computationally expensive, since all samples of the event interval have to be collected and incorporated into the Gaussian function.

6) CrossCorrelationRSSI: Another approach using all available RSSI samples per link is the Sliding Dot Product, also known as cross correlation. The cross correlation originates in the field of signal processing. Two signals are shifted towards each other along the $\mathrm{x}$-axis, while in each step their data points are multiplied with each other. The resulting product is called the correlation coefficient $\operatorname{coef}_{c c}$. The higher the correlation coefficient is, the more similar are the data streams. This method is often used to measure how far the signals need to be shifted in order for them to reach their point of highest similarity.

In this paper we use the cross correlation to sample-wise compare the data streams of two links by computing their $\operatorname{coef}_{c c}$. Given the characteristic of the input data, the highest similarity is obtained when the events of both links are overlapping.

The approach works in detail as follows: The first sample of the first link is multiplied with the last sample of the second link. The resulting cross correlation coefficient is stored. Next the links are shifted towards each other by increasing the number of samples that are compared. The first two samples of the first link are compared with the last two of the other and again the correlation coefficient is stored. This is repeated until the last sample of the first link has been compared with the first sample of the last link. Afterwards, the highest correlation coefficient between the links is selected and the number of shifts to reach this coefficient is counted.

Since the frequency with which the samples are sent, is known, the number of shifts indicates how many samples have 


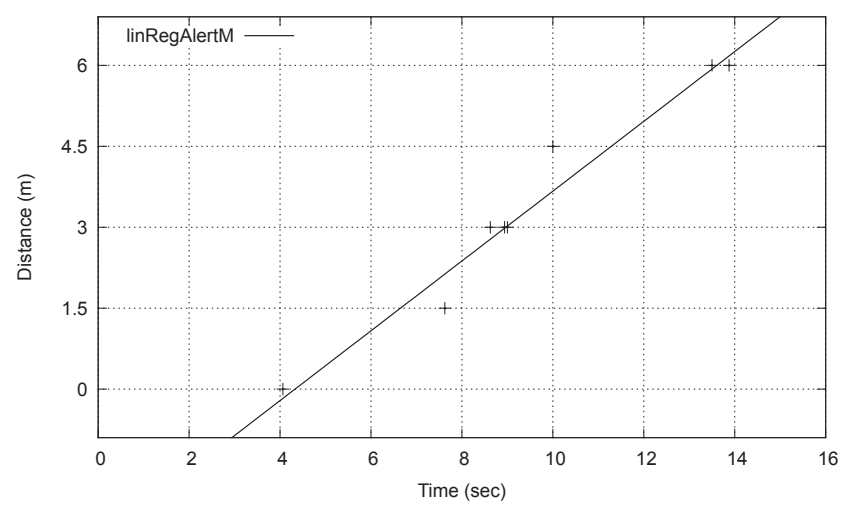

Fig. 3. Linear regression using alert magnitude values of five links

been sent in the time it took the target to cross the space between first and second link. Analysing the sample rate we deduce the time between the LoS crossings. Strictly speaking, the cross correlation is not searching for the best sample to describe the event, but computes the velocity directly.

\section{B. Alert Magnitude based Techniques}

All unaltered RSSI-based techniques work best with antifading links, since they use the minimum RSSI value and on these links the maximum drop in the RSSI level is characterizing the crossing event. In the case of deep-fading links, where the signal improves after a target enters the link, a different method is needed. On those links the RSSI values near the peak are higher, even though fluctuations cause some RSSI samples to have a very low value.

To handle both cases, a RSSI variance based technique is used. Contrary to the absolute value, the variation of the RSSI always increases when a target is in the link [16]. In this paper the algorithm from [4] is applied. While the better handling of the issue of anti- and deep-fading links is addressed by changing the input data, the general issue of selecting a sample still remains the same. This being the case, the aforementioned methods of finding the peak of the LoS crossing event can also be applied on the alert magnitude.

All techniques using the alert magnitude are summarized in the second column of Table I.

1) maxAlertM: Following the given nomenclature in [4], the technique searching for the maximum alert magnitude is called maxAlertM. After all samples with the highest alert magnitude are found, the first occurring sample, which is the sample with the minimum timestamp, is selected.

2) medianAlertM: Like the medianRSSI approach, the medianAlertM approach selects the middle one of all samples with the highest alert magnitude. If an even number of samples with the same highest alert magnitude is encountered, the first occurring of the two middle samples is selected as a tiebreaker.

3) avgAlertM: The avgAlertM approach computes the average time from the timestamps of the collected samples with

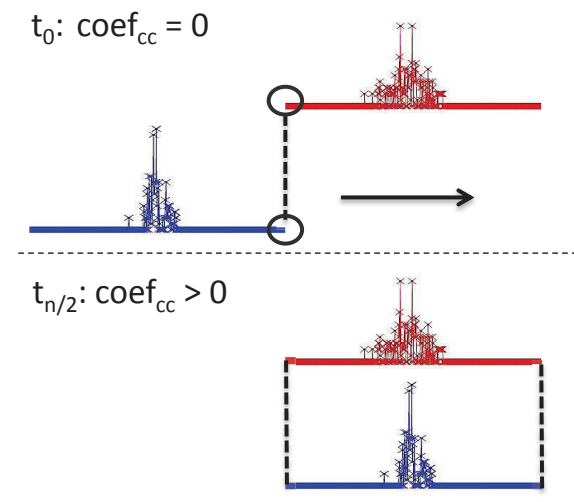

Fig. 4. General mechanism behind the cross correlation

the highest alert magnitude. Again, this is analogous to the RSSI-based approach, in this case avgRSSI.

4) linRegAlertM: The approach to compute the linear regression, based on the alert magnitude, is LinRegAlertM. It is computed from all samples with the maximum alert magnitude of each link, using data from all available links. The resulting regression line is visualized as an example in Fig. 3. The displayed data is taken from the first run of the first measurement of our experiment. The y-axis value represents the distance of a radio link to the first link of the experiment set-up, the $\mathrm{x}$-axis value represents the arrival time of the samples. For this example the distance between the links was set to $1.5 \mathrm{~m}$ and samples from five different links are used.

5) CurveFitting: While RSSI values are always unsteady and slightly fluctuating, the alert magnitude takes the form of a steady line, except during an event. All values are zero, as long as the link is not disturbed. However, they become greater than zero when indicating a target in the area of the radio link. This behaviour is beneficial to the CurveFitting approach, which fits a Gaussion function to all available alert magnitude values. Again, the precise time of the peak is not restricted by the timestamp of the samples.

Since the alert magnitude is based on the RSSI variance, this more closely resembles the curve fitting used in [13], then CurveFittingRSSI does.

6) CrossCorrelation: Computing the cross correlation from the alert magnitude values is the CrossCorrelation approach. The mechanism behind this approach is visualized in Fig. 4. In the beginning of the correlation process $\operatorname{coef}_{c c}$ equals 0 , but is gradually increased, when the data streams of the alert magnitude are shifted towards each other.

\section{Alert Magnitude Set based Techniques}

To enhance the performance of the methods using the alert magnitude and to take precautions against outliers, we introduce a new group of techniques. Instead of only searching for the samples with the maximum alert magnitude, the set 


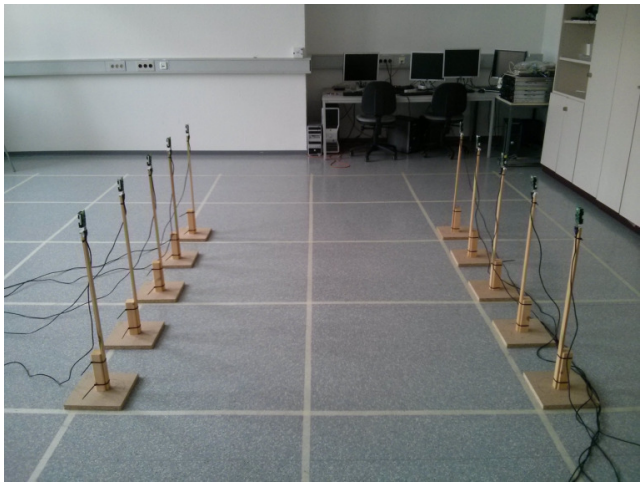

Fig. 5. Gird of motes in an lab environment

of $n$ samples with the highest values is collected. The value of $n$ is analysed further in section IV. The set includes a guaranteed minimum number of $n$ sample, but can include an even greater number, when more samples with the same high alert magnitude exist. In this case all samples are added to the set. Using a set of samples reduces the effect of the sample with the maximum alert magnitude being an outlier and thus unfavourable influencing the result.

Since the input data has been reduced to a selective subset of the complete data stream, the curve fitting and cross correlation techniques cannot be used. However, most methods estimating the velocity from the highest alert magnitude value can also be applied to this slightly enlarged set of samples.

1) topMaxAlertM: The topMaxAlertM approach uses the first occurring sample of the set with the $n$ highest values. This could be realized by either selecting the first occurring sample with the highest alert magnitude, or the first occurring sample of the complete sample set. However, the first case is identical to the maxAlertM approach, in the second case a timestamp from the border of the event is selected, not representative for the peak.

Because of the above mentioned issues, the topMaxAlertM approach is not further considered in the evaluation.

2) topMedianAlertM: The topMedianAlertM approach selects the middle sample of collected set. This sample does not necessarily have to be the one with the highest value.

3) topAvgAlertM: The topAvgAlertM technique uses the timestamps of all samples in the collected set to estimate the time of the peak by computing the average time, again loosening the restriction of the precise timestamp.

4) topLinRegAlertM: topLinRegAlertM models the linear regression using the slightly larger data set, again with input from multiple links.

\section{Evaluation}

For the evaluation of the different techniques we perform an experiment with a test set-up, as seen in Fig. 5. The set-up consists of ten Crossbow telosB sensor nodes, five on each side of a $2 \mathrm{~m}$ wide and $6 \mathrm{~m}$ long detection area. The motes are set in an interval of $1.5 \mathrm{~m}$ on the same side. Radio links are formed between the motes directly opposite of each other in a

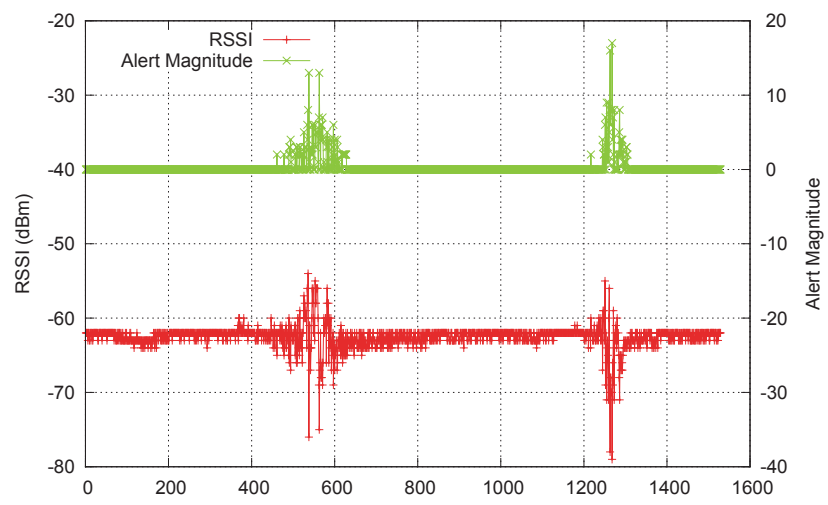

Fig. 6. Example of recorded RSSI and alert magnitude

way, that five consecutive links are generated across the space. During the experiment the links exhibited a mean RSSI of $62.5,-57.9,-58.3,-64.5$ and -60.26 respectively from link one to link five. The motes use the TI MSP430 microcontroller and the CC2420 transceiver chip. They are sending on a frequency of $2.4 \mathrm{GHz}$ and use a TDMA scheme with a 62.5 millisecond cycle time to avoid collisions. This value is chosen as a trade-off between energy consumption and detection accuracy. For our analysis all sent messages are recorded as samples using the IRIS tool introduced in [17]. IRIS is an experiment management tool, that allows for simultaneous data collection and visualisation. Samples collected can be analysed using provided or self-written functions and can be saved for further processing.

To evaluate the accuracy of the velocity estimation of a moving target, we compute the average error respective to the actual velocity. Two measurements with 12 runs each are performed in an lab environment. The error values are calculated as an average of the 12 runs for each measurement. During one experiment run, the target is entering the lab, walking crossing the links through the detection area once and then leaves the room. For the purpose of this evaluation, we assume that the target is walking with constant speed, does not suddenly change direction and walks directly in the middle of the detection area in a straight line. In the first measurement the target is walking with a speed of $0.6 \mathrm{~m} / \mathrm{s}$, in the second measurement with $1.35 \mathrm{~m} / \mathrm{s}$. The behaviour of the RSSI and the corresponding alert magnitude can exemplary be seen in Fig. 6. The figure shows a target crossing a link first with the slower, then with the faster speed. Markings on the floor indicating the step interval and a metronome to time the steps are used to help the target maintain constant speed, while still walking normally. Also, to not be reliant on the human perception of time, the precise moment of the LoS crossing is recorded as ground truth, using a laser based system.

The results of the measurements are summarized in Table II and Table III. Table II contains the average error in \% and its Sample Standard Deviation. Column one and two list the values for the measurements with the slower speed of 
TABLE II

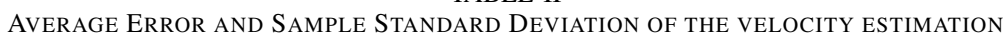

\begin{tabular}{|c|c|c|c|c|c|}
\hline \multirow{2}{*}{\multicolumn{2}{|c|}{ Technique }} & \multicolumn{2}{|c|}{ Measurement Velocity $0.6 \mathrm{~m} / \mathrm{s}$} & \multicolumn{2}{|c|}{ Measurement Velocity $1.35 \mathrm{~m} / \mathrm{s}$} \\
\hline & & Average Error (\%) & Sample Standard Deviation & Average Error (\%) & Sample Standard Deviation \\
\hline 1 & $\operatorname{minRSSI}$ & 16.77 & 13.40 & 11.15 & 6.82 \\
\hline 2 & medianRSSI & 17.25 & 13.97 & 11.50 & 6.91 \\
\hline 3 & avgRSSI & 16.18 & 13.94 & 11.33 & 6.84 \\
\hline 4 & linRegRSSI & 12.79 & 10.95 & 11.03 & 7.77 \\
\hline 5 & CurveFittingRSSI & 17.10 & 23.15 & 27.00 & 20.40 \\
\hline 6 & CrossCorrelationRSSI & 83.99 & 1.64 & 86.98 & 1.59 \\
\hline 7 & maxAlertM & 12.03 & 12.15 & 11.59 & 8.34 \\
\hline 8 & medianAlertM & 11.41 & 12.57 & 12.16 & 8.16 \\
\hline 9 & avgAlertM & 11.72 & 12.34 & 11.88 & 8.22 \\
\hline 10 & linRegAlertM & 7.00 & 6.21 & 13.21 & 15.91 \\
\hline 11 & CruveFitting & 12.03 & 12.15 & 11.59 & 8.34 \\
\hline 12 & CrossCorrelation & 5.04 & 3.40 & 7.17 & 4.04 \\
\hline 13 & topMedianAlertM 5 & 4.12 & 3.83 & 6.16 & 5.04 \\
\hline 14 & topAvgAlertM 5 & 4.12 & 4.30 & 3.82 & 4.16 \\
\hline 15 & topLinRegAlertM 5 & 21.73 & 13.32 & 22.76 & 23.45 \\
\hline
\end{tabular}

$0.6 \mathrm{~m} / \mathrm{s}$, column three and four the values for the faster speed of $1.35 \mathrm{~m} / \mathrm{s}$. Each row shows the results for a different technique. Table III contains the results of the measurement runs with the maximum error for each approach and the Squared Mean Error, also for both measurements and velocities.

\section{A. RSSI}

The results of the measurements using the minimum RSSI data stream, minRSSI, can be found in the first row of Table II, for both slower and faster speed. Estimating the velocity from the first found sample selected by the minRSSI has an average error of $16.8 \%$ with a sample standard deviation of 13.4 for the slow velocity measurement and $11.2 \%$ error with a deviation of 6.8 for the faster velocity. This corresponds to an absolute error in the estimation of $0.1 \mathrm{~m} / \mathrm{s}$ for a speed of $0.6 \mathrm{~m} / \mathrm{s}$ and an absolute error of $0.15 \mathrm{~m} / \mathrm{s}$ for a speed of $1.35 \mathrm{~m} / \mathrm{s}$.

Achieving a high accuracy using this technique turns out to be easier when detecting a target walking in a faster pace. The duration of the LoS crossing event is shorter, involving fewer samples and a narrower time interval, as seen in Fig. 6.

Using the median of all samples with the minimum RSSI (medianRSSI) or the average of those (avgRSSI) does not improve the performance significantly. The deep-fade link behaviour is ignored, which leads to a less accurate event detection and higher error values.

The most accurate approach according to our results, when working with pure RSSI values, is computing a linear regression. linRegRSSI shows a slightly better performance in the measurement with slower speed, and average performance, when the target is moving faster. Since multiple links are used in this approach, irregularities of one link can be evened out by the others.

Modelling the stream of RSSI values with a Gaussian function in the CurveFittingRSSI approach is unfavourable compared to the other techniques. Since the RSSI varies around a baseline, creating both positive and negative spikes in the case of an event, CurveFittingRSSI has a very high sample standard deviation. This causes very inaccurate behaviour.

Comparing two RSSI data streams using a cross correlation is possible, but since the similarity of two links is compared, the original input data needs to be similar. The unsteady and fluctuating nature of the RSSI with a high variance during the crossing event prevents this. Also, the deep-fading and anti-fading behaviour of the RSSI creates completely different characteristics, that cannot directly be compared. The resulting error values are constantly above $80 \%$ in both our measurements and have a very small Sample Standard Deviation, achieving in the worst performance of all tested RSSI-based methods.

\section{B. Alert Magnitude}

1) maxAlertM, medianAlertM and averageAlertLevel: Using the alert magnitude as a basis for the detection achieves better results for the slow velocity measurement and similar results to the RSSI-based techniques for faster speeds. Exploitation of the RSSI variance, and by doing so coping with the deep-fade qualities of the links, is the cause of this improvement. The average estimation error of maxAlertM can be seen in row seven of Table II, the results for medianAlertM and averageAlertLevel are in row eight and nine. All three techniques perform similar to each other, with error values around $11.5 \% \pm 1 \%$, which is an improvement of about $4 \%$ towards the RSSI-based techniques in the $0.6 \mathrm{~m} / \mathrm{s}$ measurement.

2) linRegAlertM: Using the linear regression has an improvement of $5 \%$ when applied to the data of the slow velocity measurement. However, linRegAlertM shows worse performance with the data of the faster velocity measurement. The average error is $13.2 \%$, which is $2 \%$ worse than when based on the RSSI. 
TABLE III

Maximum and SQuared Mean ERror of the Velocity estimation

\begin{tabular}{|l|l|c|c|c|c|}
\hline \multirow{2}{*}{\multicolumn{2}{|c|}{ Technique }} & \multicolumn{2}{|c|}{ Measurement Velocity 0.6m/s } & \multicolumn{2}{c|}{ Measurement Velocity 1.35m/s } \\
\cline { 2 - 6 } & Maximum Error (\%) & Squared Mean Error & Maximum Error (\%) & Squared Mean Error \\
\hline 1 & minRSSI & 51.89 & 460.85 & 28.07 & 170.74 \\
3 & medianRSSI & 51.89 & 492.89 & 28.07 & 179.94 \\
4 & avgRSSI & 51.89 & 455.93 & 28.07 & 175.09 \\
5 & linRegRSSI & 39.45 & 283.59 & 26.85 & 181.98 \\
6 & CurveFittingRSSI & 85.23 & 828.11 & 69.77 & 1144.93 \\
\hline 7 & CrossCorrelationRSSI & 87.15 & 7057.48 & 91.27 & 7567.14 \\
8 & maxAlertM & 46.85 & 292.43 & 32.73 & 203.99 \\
9 & avgAlertM & 46.856 & 288.19 & 32.73 & 214.34 \\
10 & linRegAlertM & 46.85 & 289.73 & 32.73 & 208.78 \\
11 & CruveFitting & 22.71 & 87.58 & 59.34 & 427.44 \\
12 & CrossCorrelation & 46.85 & 292.43 & 32.73 & 203.99 \\
\hline 13 & topMedianAlertM & 11.36 & 36.88 & 14.71 & 67.68 \\
14 & topAvgAlertM & 12.77 & 31.61 & 17.74 & 63.30 \\
15 & topLinRegAlertM & 18.12 & 35.53 & 15.87 & 31.94 \\
\hline
\end{tabular}

Since the target is moving with a higher speed, the time between the LoS crossing of the links is shorter. This has an impact on the slope of the linear regression line. It is more steep and outliers have a higher influence since fewer samples are sent, explaining the behaviour in the faster velocity measurement.

3) Curve Fitting: Curve fitting is performed using all values of the respective data streams, without previous filtering for minimum RSSI or maximum alert magnitude values. Fitting a curve through the alert magnitude values achieves similar results to maxAlertM, medianAlertM and avgAlertM, as seen in row 11 of Table II. The average error is at $12 \%$ for the first measurement and at $11,6 \%$ for the second.

The results of the alert magnitude based CurveFitting achieve a higher accuracy than fitting a curve to the RSSI data stream. The steady nature of the alert magnitude, which is only interrupted in the case of a detection event, positions the maximum of the Gaussian function close to the peak of the LoS crossing. Still, the results are on the same accuracy level as maxAlertM, medianAlertM and averageAlertLevel.

4) Cross Correlation: The cross correlation computes the correlation coefficient $\operatorname{coef}_{c c}$ between two links. To utilize the approach and estimate the velocity of a target, all possible link combinations for both measurements are analysed first. The results of this test are shown in Fig. 7. There, the average error is plotted over the distance of the links towards each other. A clear trend is visible, showing that the estimation becomes more accurate, the further the links are apart. The overall error is being reducing and the minimum and maximum error values are closer to the average. The messages for the experiment are being sent with a frequency of 62.5 milliseconds and it takes the target a certain time to cross the distance between two links. The larger the distance, the longer is the time needed to cross it and the more samples can be sent in the duration,

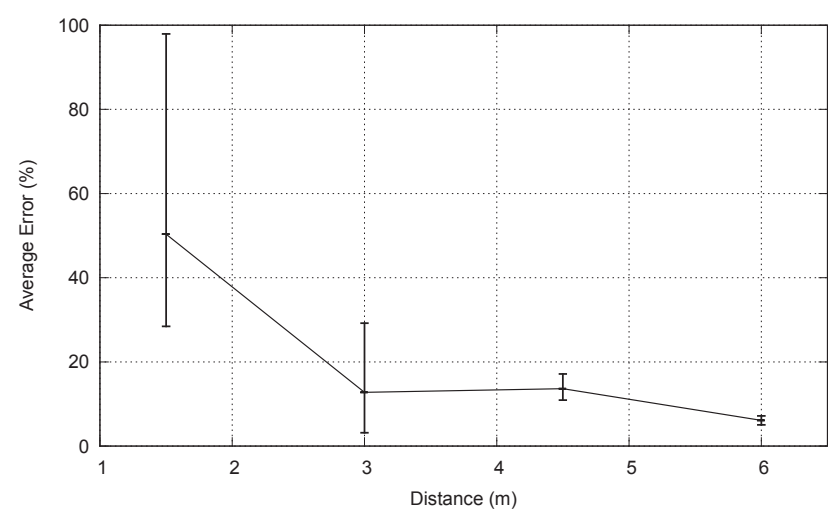

Fig. 7. Average of the velocity estimation error over the link distance

explaining the increase in the accuracy. For this reason the cross correlation between the two links farthest apart is used for the comparison with the other techniques.

As mentioned in Section IV-A, estimating the velocity using the cross correlation of the RSSI values has a very low accuracy, because of the RSSI fluctuations. However, applying it on the alert magnitude is very promising. The average error is shown in row 12 of Table II. It is at $5.0 \%$ with a sample standard deviation of 3.4 for the slow velocity measurement. For the fast velocity measurement these values are at $7.2 \%$ average error with 4.0 sample standard deviation.

These results surpass the previous techniques using the maximum alert magnitude. Since the alert magnitude values are zero except during an event, as seen in Fig. 6, the correlation coefficient reaches its maximum when the events on two different links are directly overlapping. This achieves a precise approximation of the time between the LoS crossing events on two compared links used in the velocity estimation. 


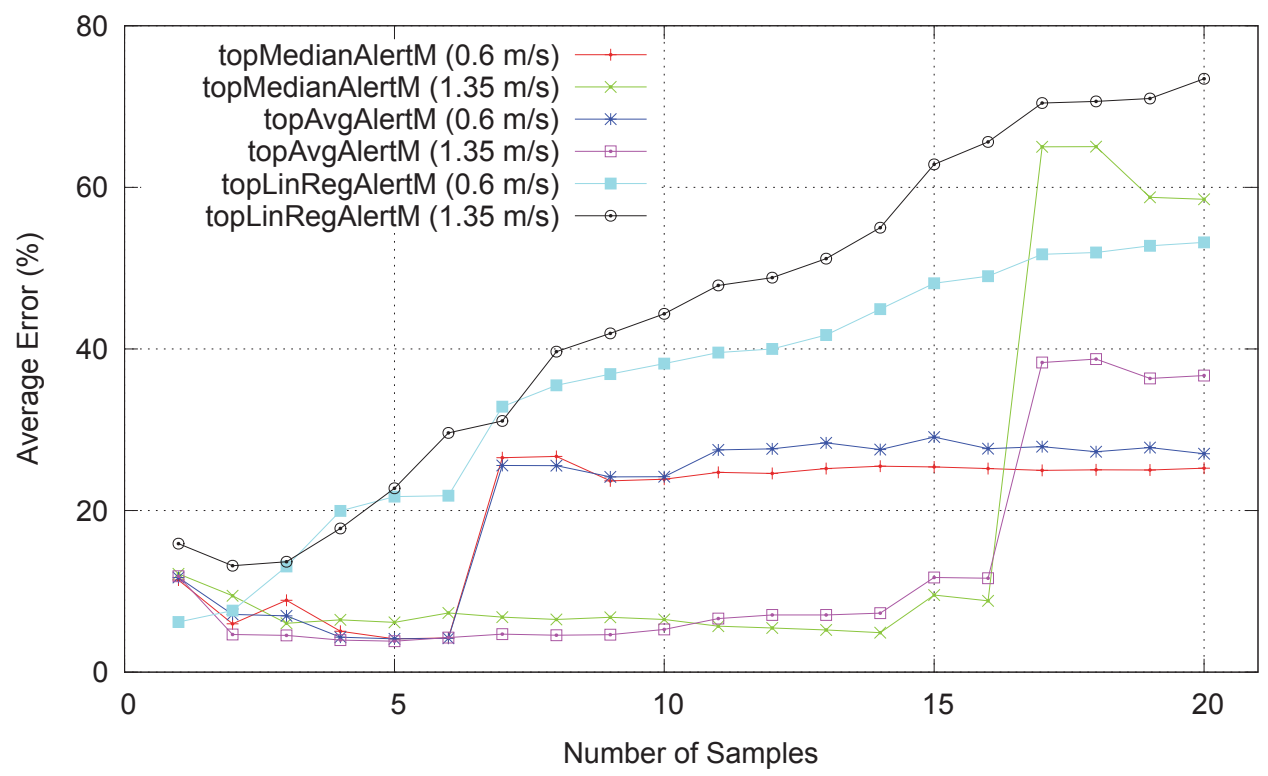

Fig. 8. Average velocity estimation errors with different sizes of alert magnitude sets

\section{Alert Magnitude Set}

Instead of using the sample(s) with the maximum alert magnitude, using the set of the $n$ highest alert magnitude values can achieve a very different result. The set provides a more balanced picture of the event, since it includes all of the most interesting samples and not just the highest, reducing the influence of outliers. Unfortunately, this is not beneficial to all methods. The CurveFitting and CrossCorrelation techniques use all the samples from the complete measurement and cannot be applied on a subset. Furthermore, since the sample with the maximum alert magnitude is still in the set of the highest $n$ samples, using the topMaxAlertM approach will not have a different outcome than maxAlertM, as explained in section III-C1. However, for the techniques of topMedianAlertM, topAvgAlertM and topLinRegAlertM the improved sample set is applicable.

The influence of the size of the sample set is analysed in Fig. 8, showing the average error over the number of samples. For each of the remaining three techniques all sample set sizes from 1 to 20 were examined. The set size of 1 equals the respective approach based on the maximum alert magnitude value. The figure displays six graphs, two for each technique, split by the velocity of the measurement. To further differentiate between the techniques an index $i$ is introduced, indicating the size of the sample set. topMedianAlertM $\mathrm{M}_{3}$, for example, would describe the topMedianAlertM approach with a sample set size of 3 samples.

The analysis shows a high improvement of the topMedianAlertM and the topAvgAlertM approach for the measurement with a velocity of $0.6 \mathrm{~m} / \mathrm{s}$, when increasing the sample size to up to 6 samples in the set. The lowest error is achieved by topMedianAlertM $\mathrm{M}_{5}$ with an average error of $4.12 \%$, directly followed by topAvgAlertM $\mathrm{M}_{5}$ with an average error of $4.124 \%$ for the slower velocity. This is an accuracy of around $95.8 \%$, estimating the velocity of the target. These improvements are due to the fact that both techniques benefit from the larger selection of possible samples describing the peak of the crossing event.

For the fast velocity measurement of $1.35 \mathrm{~m} / \mathrm{s}$ the topAvgAlertM $\mathrm{M}_{5}$ approach achieves the lowest average error of $3.82 \%$, giving it an estimation accuracy of $96.1 \%$. For the topMedianAlertM techniques topMedianAlertM $M_{1} 4$ has the lowest error with $4.88 \%$.

Additionally, Fig. 8 indicates a trade-off between too few or too many samples in the sample set. While the performance of the topMedianAlertM and topAvgAlertM techniques initially improves when adding samples to the set, the average error abruptly increases after a certain threshold. For the slow velocity measurement this happens in our experiment at sample set size 7 , for the fast velocity measurement at sample set size 17.

The reason for this is the inclusion of samples in the set which are not representative for the peak. These sample cause a reduction in precision. The effect occurs, when the number of samples with unique values is not very large. If only a few samples with high alert magnitude values exist, all of them will be included into the sample set. However, when raising the set size, more samples will need to be added. Eventually, some samples with only moderate alert magnitude values will be in the sample set. Those are less likely to describe the detection event. As soon as that happens, the accuracy of the alert magnitude set based techniques diminishes.

Table II summarizes the results for topMedianAlertM $M_{5}$ in row 13, and topAvgAlertM ${ }_{5}$ in row 14.

Since more samples are guaranteed to be in the sample set, the linear regression loses much of its accuracy. Fig. 8 
shows: The more samples are added to the set, the worse the topLinRegAlertM technique performs. This deterioration occurs, because the values of all samples are included in the computation. The average error drops from 7\% (linRegAlertM) to $21,7 \%$ (linRegAlertM ${ }_{5}$ ) in the slow velocity measurement and from $13,2 \%$ to $22,8 \%$ in the fast velocity measurement. The results for linRegAlertM ${ }_{5}$ are listed in Table II, row 15 .

The evaluation shows that the topMedianAlertM ${ }_{5}$ and the topAvgAlertM $\mathrm{M}_{5}$ techniques are the best choices for accurately detecting the peak of an event and estimating the velocity of a target. Furthermore, they are also less computational expensive then a linear regression, the curve fitting technique or the cross correlation.

\section{CONCLUSION \& Future WORK}

In this paper we experimentally evaluated 15 techniques in order to estimate the velocity of a target moving through a set of radio links. The precise moment of the Line of Sight crossing is determined based on the collection of RSSI samples. The techniques are separated into groups, based on their input values. Analysing the average of the minimum RSSI attains less accurate results than focusing on the maximum alert magnitude or the set of the $n$-highest alert magnitude samples. This is caused by the RSSIs unstable character.

Three techniques were found delivering unsuitable results below $80 \%$ accuracy, nine techniques performed moderately, three techniques achieved a performance above $90 \%$ estimation accuracy. Performing a cross correlation of the links with the highest distance from each other achieves good results in estimating the velocity. The accuracy for slow moving targets is around $95.0 \%$, for fast moving targets it is around $92.9 \%$. Furthermore, not focusing on the samples with the highest absolute value, but on the set of highest values offers a layer of protection against outliers and improves simple median or average computing techniques. Selecting the median timestamp of the set of samples with the highest detection value achieves $95.8 \%$ accuracy for slow moving targets and $95.1 \%$ for fast moving ones. Computing the average of those samples also achieves $95.8 \%$ accuracy for slow moving targets and $96.1 \%$ accuracy for fast ones.

In the future we want to extend our research by using the velocity estimation to differentiate between multiple targets in the same radio environment.

\section{REFERENCES}

[1] K. Woyach, D. Puccinelli, and M. Haenggi, "Sensorless sensing in wireless networks: Implementation and measurements," 2006 4th International Symposium on Modeling and Optimization in Mobile, Ad Hoc and Wireless Networks, WiOpt 2006, 2006. doi: 10.1109/WIOPT.2006.1666495. Available: http://dx.doi.org/10. 1109/WIOPT.2006.1666495

[2] S. Hussain, R. Peters, and D. Silver, "Using received signal strength variation for surveillance in residential areas," SPIE Defense ..., 2008. Available: http://proceedings.spiedigitallibrary.org/ proceeding.aspx ?articleid $=837114$
[3] J. Wilson and N. Patwari, "Radio tomographic imaging with wireless networks," Mobile Computing, IEEE Transactions on, vol. 9, no. 5, pp. 621-632, 2010. Available: http://ieeexplore.ieee.org/xpls/abs\{\\}all.jsp? arnumber $=5374407$

[4] O. Kaltiokallio and M. Bocca, "Real-Time Intrusion Detection and Tracking in Indoor Environment through Distributed RSSI Processing," 2011 IEEE 17th International Conference on Embedded and Real-Time Computing Systems and Applications, pp. 61-70, aug 2011. doi: 10.1109/RTCSA.2011.38. Available: http://ieeexplore.ieee.org/lpdocs/ epic03/wrapper.htm?arnumber $=6029830$

[5] M. Kochláň, J. Miček, and P. Ševčík, "2.4ghz ism band radio frequency signal indoor propagation," in Proceedings of the 2014 Federated Conference on Computer Science and Information Systems, ser. Annals of Computer Science and Information Systems, M. P. M. Ganzha, L. Maciaszek, Ed., vol. 2. IEEE, 2014. doi: 10.15439/2014F299 pp. pages 1027-1034. Available: http://dx.doi.org/10.15439/2014F299

[6] J. Yang, Y. Ge, and H. Xiong, "Performing Joint Learning for Passive Intrusion Detection in Pervasive Wireless Environments," .. 2010 Proceedings IEEE, 2010. Available: http://ieeexplore.ieee.org/ xpls/abs $\left\{\_\right\}$all.jsp?arnumber $=5462148$

[7] M. Bocca, O. Kaltiokallio, N. Patwari, and S. Venkatasubramanian, "Multiple target tracking with rf sensor networks," IEEE Transactions on Mobile Computing, vol. 13, no. 8, pp. 1787-1800, 2014. doi: 10.1109/TMC.2013.92. Available: http://dx.doi.org/10.1109/TMC.2013. 92

[8] F. Adib, Z. Kabelac, D. Katabi, R. C. Miller, I. Nsdi, and Z. Kabelac, "Multi-Person Localization via RF Body Reflection," Usenix Nsdi, 2014 Available: https://www.usenix.org/conference/nsdi15/technical-sessions/ presentation/adib

[9] T. Li, Y. Wang, L. Song, and H. Tan, Wireless Sensor Networks: 12th European Conference, EWSN 2015, Porto, Portugal, February 9-11, 2015. Proceedings. Cham: Springer International Publishing, 2015 , ch. On Target Counting by Sequential Snapshots of Binary Proximity Sensors, pp. 19-34. ISBN 978-3-319-15582-1. Available: http://dx.doi.org/10.1007/978-3-319-15582-1_2

[10] J. Wilson and N. Patwari, "See-through walls: Motion tracking using variance-based radio tomography networks," Mobile Computing, IEEE Transactions on, vol. 10, no. 5, pp. 612-621, 2011. Available: http://ieeexplore.ieee.org/xpls/abs $\{\backslash$ \}all.jsp?arnumber $=5582100$

[11] O. Kaltiokallio, M. Bocca, and N. Patwari, "Follow @grandma: Longterm device-free localization for residential monitoring," Proceedings - Conference on Local Computer Networks, LCN, pp. 991-998, 2012. doi: 10.1109/LCNW.2012.6424092. Available: http://dx.doi.org/ 10.1109/LCNW.2012.6424092

[12] J. Wilson and N. Patwari, "A Fade-Level Skew-Laplace Signal Strength Model for Device-Free Localization with Wireless Networks," IEEE Transactions on Mobile Computing, vol. 11, no. 6, pp. 947-958, jun 2012. doi: 10.1109/TMC.2011.102. Available: http //ieeexplore.ieee.org/lpdocs/epic03/wrapper.htm?arnumber=6188339

[13] N. Kassem, A. E. Kosba, and M. Youssef, "RF-Based Vehicle Detection and Speed Estimation," 2012 IEEE 75th Vehicular Technology Conference (VTC Spring), pp. 1-5, may 2012. doi: 10.1109/VETECS.2012.6240184. Available: http: //ieeexplore.ieee.org/lpdocs/epic03/wrapper.htm?arnumber=6240184

[14] O. Karpis, "Sensor for vehicles classification." in FedCSIS, 2012, pp. 785-789. Available: https://fedcsis.org/proceedings/2012/pliks/215.pdf

[15] O. Kaltiokallio, "Intrusion Detection Based on Embedded Processing of Received Signal Strength Indicator," Master's thesis, Aalto University, 2011.

[16] D. Zhang and L. M. Ni, "Dynamic clustering for tracking multiple transceiver-free objects," 2009 IEEE International Conference on Pervasive Computing and Communications, pp. 1-8, mar 2009. doi: 10.1109/PERCOM.2009.4912777. Available: http://ieeexplore.ieee.org/ lpdocs/epic03/wrapper.htm?arnumber=4912777

[17] R. Figura, M. Ceriotti, C.-Y. Shih, M. Mulero-Pâzmâny, S. Fu, R. Daidone, S. Jungen, J. J. Negro, and P. J. Marrôn, "Iris: Efficient visualization, data analysis and experiment management for wireless sensor networks," EAI Endorsed Transactions on Ubiquitous Environments, vol. 14, no. 3, 11 2014. doi: 10.4108/ue.1.3.e4. Available: http://dx.doi.org/10.4108/ue.1.3.e4 